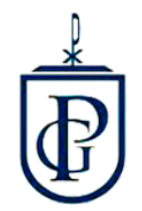

Veritas Et Scientia

Vol. $7, N^{\circ} 1,809-815$

Enero - Junio del 2018.

ISSN $2307-5139$

\title{
SISTEMA DE FONDOS COLECTIVOS COMO ESTRATEGIA PARA LA ADQUISICIÓN DE ACTIVOS DE LAS EMPRESAS DE TACNA EN EL 2016
}

SYSTEM OF COLLECTIVE FUNDS AS A STRATEGY FOR THE ACQUISITION OF ASSETS OF TACNA COMPANIES IN 2016

Joanette Ivette Vargas Monroy ${ }^{1}$

Presentado: 09/12/2017

Aceptado: 04/03/2018

Publicado online: 11/06/2018

\section{RESUMEN}

El Este trabajo de investigación reúne los diferentes aspectos relacionados al Sistema de Fondos Colectivos como estrategia para la adquisición de activos de las de las empresas de Tacna en periodo 2016, así mismo se tiene que el Sistema de Fondos Colectivos es una forma de financiamiento que con el tiempo ha venido adquiriendo mayor relevancia como una opción para la adquisición de activos. Es no experimental, descriptivo y correlacional. Se ha considerado como población a las empresas financiadas con fondos colectivos de la ciudad de Tacna, como técnica se ha utilizado una encuesta y como instrumento se utilizó un cuestionario para evaluar las variables objeto de estudio. El área de investigación está enmarcada en la Contabilidad Financiera. Las Empresas Administradoras de Fondos Colectivos, vienen siendo una alternativa de financiamiento para la adquisición de activos para las empresas de Tacna, permitiendo mejorar sus ingresos y por ende el crecimiento económico financiero de cada una de estas empresas, permitiéndoles mejorar su rentabilidad y por ende su desarrollo económico, dando oportunidad a miles de personas a acceder a mejores niveles y calidad de vida.

Palabras Clave: Fondos colectivos, estrategias, adquisición de activos.

\section{ABSTRACT}

This research work brings together the different aspects related to the Collective Funds System as a strategy for the acquisition of assets from the Tacna companies in 2016, as well as the Collective Funds System is a form of financing that time has been acquiring greater

\footnotetext{
${ }^{1}$ Magíster en Contabilidad, Tributación y Auditoria
} 
relevance as an option for the acquisition of assets. It is non-experimental, descriptive and correlational. The companies financed with collective funds from the city of Tacna have been considered as a population, as a technique a survey has been used and as a tool a questionnaire was used to evaluate the variables under study. The research area is framed in Financial Accounting. The Collective Funds Management Companies have been an alternative financing for the acquisition of assets for the Tacna companies, allowing to improve their income and therefore the economic and financial growth of each of these companies, allowing them to improve their profitability and therefore their economic development, giving thousands of people the opportunity to access better levels and quality of life.

Keywords: Collective funds, strategies, asset acquisition.

\section{INTRODUCCIÓN}

Las empresas del Perú vienen formando el brazo productivo del país, ya que apuntan a su consolidación como promotor del crecimiento y del desarrollo económico; de alguna manera da oportunidad a miles de personas a acceder a mejores niveles de vida. Las empresas adolecen de una serie de deficiencias estructurales, que no se han podido corregir y que no permiten potenciarla a su máxima capacidad competitiva, como el capital propio que no satisface sus necesidades para cubrir su capital de trabajo así como de inversión; además las entidades financieras inclinan su posición financiera hacia las grandes y medianas empresas, teniendo algunas empresas una mínima participación en su cartera de colocaciones y en caso que se otorgue el financiamiento, ofrecen créditos cuyo costo financiero es bastante elevado y que no permiten que las empresas puedan acceder a dicho financiamiento. El sistema de fondos colectivos es una forma de financiamiento que con el tiempo ha venido adquiriendo mayor relevancia como una opción para la adquisición de bienes y servicios. De esta forma, se constituye en un esquema alternativo a los créditos otorgados por los bancos y otras instituciones financieras. Las administradoras de fondos colectivos vienen siendo una alternativa de financiamiento para la adquisición de activos de estas empresas, permitiendo mejorar sus ingresos y por ende el crecimiento económico financiero.

Salazar, O. (2010), concluye que es factible realizar un fondo colectivo de desarrollo inmobiliario en la ciudad de Quito, que la administradora de fondos Pichincha S.A. ya que cumple con el capital necesario, tiene una estructura organizacional adecuada y en el aspecto legal están dentro de los parámetros exigidos por la ley de Mercado y Valores, brindándole una rentabilidad adecuada. Manrique, J. (2013), afirma que el ahorro institucional captado a través de los fondos mutuos, compañías de seguros y fondos privados de pensiones se ha consolidado en los últimos años; y que los fondos mutuos permiten a las empresas a obtener financiamiento a un costo más asequible que el del sistema bancario tradicional, para el mejoramiento de su competitividad. Imbaquingo, K (2008) refiere que es importante que una empresa cuente con un inventario de activos fijos completo y actualizado, que permita al área encargada una mejor administración y control de los activos durante su vida útil. Isa N., Romani F., Romani J. (2013) concluye que los fondos de inversión es una manera eficiente de financiamiento que en sistema bancario convencional para mediana empresa. Según Hernández D. (2015), los Fondos Colectivos consisten en formar grupos cerrados de personas sean jurídicas o naturales, para que hagan aportaciones en forma mensual por un periodo determinado a fin de contar con un fondo común, adquirir bienes o servicios objeto del contrato. En un Sistema de Fondos Colectivos cada uno de los participantes adquiere un bien o servicio aportando cierto número de cuotas pagando periódicamente, el asociado paga el valor correspondiente a su cuota mensual más un pago único de costo de inscripción por derecho de ingreso a un grupo. (Hernández D. 2015, p.06). La adjudicación de lo adquirido se realiza mensualmente entre los participantes que se encuentren al día 
con sus aportes de cuotas y que aún no hayan sido adjudicados. Consiste en realizar una entrega aleatoria mensual entre todos los asociados solventes de cada grupo. (Hernández D. 2015, p.09). Una vez adjudicado el bien, el asociado constituirá las garantías a satisfacción del grupo y se procederá a la entrega definitiva del bien o servicio. Lira J (2016), indica que los Fondos Colectivos pueden ser una buena alternativa de financiamiento y por ende rentable. El Reglamento de Fondos Colectivos (2014), normará el sistema de fondos colectivos y las empresas administradores deberán sujetarse a esas normas así como las personas que se relacionan directa o indirectamente con las referidas empresas, (Resolución SMV Nº 020-2014-SMV/01, 2014). La Comisión Nacional Supervisora de Empresas y Valores - CONASEV, es la institución encargada de supervisar y controlar a las empresas administradoras de fondos colectivos. Toda esa referencia a CONASEV, debe entenderse hecha a la Superintendencia del Mercado de Valores-SMV. El 28 de julio se publicó la Ley 29782 Ley de Fortalecimiento de la Supervisión del Mercado de Valores, mediante la cual se transforma a CONASEV en una Superintendencia. Los contratos estipulan la cancelación del saldo, la cuota inicial, y demás formalidades para definir responsabilidades de las partes contrayentes (empresa administradora y asociados). Según Effio F (2017) La norma contable que regula el tratamiento de los inmuebles, maquinaria y equipo es la NIC 16. Los principales problemas que presenta el reconocimiento contable de propiedad, planta y equipo son la contabilización de los activos, la determinación de su importe en libros y los cargos por depreciación y pérdidas por deterioro que deben reconocerse con relación a los mismos. (Effio F 2017, p. 06). Se entiende que los costos iniciales vienen a ser el valor de compra del bien más los gastos de embalaje y transporte; lo que se registra contablemente para posteriormente una vez que el bien entre en uso proceder a la provisión por depreciación para su reemplazo posterior con el transcurrir del tiempo. Asimismo, se tiene que los costos de mantenimiento deben de formar parte de los activos para poder aumentar su vida útil. Los componentes del costo, son todos los costos incurridos por una entidad en la compra o costo de construcción y todos aquellos desembolsos necesarios para colocar en condiciones de ser usados a los activos fijos. (Montenegro J., 2013, p 36).

\section{OBJETIVOS}

1. Evaluar en qué medida los fondos colectivos influyen en el beneficio económico de las empresas de la ciudad de Tacna, para la adquisición de activos fijos.

2. Analizar de qué manera los fondos colectivos influyen en los flujos de efectivo de las empresas de la ciudad de Tacna, para facilitar las compras generando un ahorro para la empresa.

3. Conocer cómo los fondos colectivos influyen en la generación de actividades productivas de las empresas de la ciudad de Tacna, para mejorar su rentabilidad.

\section{METODOLOGÍA}

Investigación aplicada, no experimental transeccional, realizada en las empresas de la ciudad de Tacna que han adquirido bien a través del sistema de fondos colectivos. La población para el presente estudio está conformada los funcionarios de las empresas de la ciudad de Tacna que han adquirido bienes a través del Sistema de Fondos Colectivos Promotora Opción S. A. EAFC.

Se diseñó un conjunto de preguntas de tipo cerrada. Los cuestionarios se aplicaron a los representantes legales de las diferentes empresas representativas, quienes proporcionaron información de cómo se está llevando el sistema de fondos colectivos de la empresa. 
Empresas de la ciudad de Tacna que han adquirido bienes a través del Sistema de Fondos Colectivos Promotora Opción S. A. EAFC

\begin{tabular}{llc}
\hline $\mathrm{N}^{\circ}$ & \multicolumn{1}{c}{ RAZÓN SOCIAL } & CONDICIÓN \\
\hline 01 & Ladrillera Jorge Martorell S.A & Remate \\
02 & Mineiro Group S.A.C. & Remate \\
03 & Martorell Becks, Daniel Alberto & Remate \\
04 & Tapia Onofre, Edgar Eduardo & Sorteo \\
05 & Asto Cabrera, Luis Cerapio & Remate \\
06 & Distribuidora Avícola Leonel E.I.R.L & Remate \\
07 & Cáceres Alarcón, Fredy Fortuna & Sorteo \\
08 & Minera \& Construcción T.Q.S.R.L. & Remate \\
09 & Autoservicios Car Wash Center E.I.R.L. & Remate \\
10 & Jhonny y Bertha S.A.C. & Sorteo \\
11 & Rengifo Díaz, Mario Edilberto & Remate \\
12 & Groumond colorantes S.A.C & Sorteo \\
13 & Quispe Huanacuni, Eloy Hernán & Remate \\
14 & KZ Constructora S.A.C. & No adjudicado \\
15 & CONECTATEC S.A.C & No adjudicado \\
16 & Comercializadora y Distribuidora & No adjudicado \\
17 & Velasco Capia, Vanesa & No adjudicado \\
18 & Grupo UROS S.A.C & No adjudicado \\
19 & Consorcio Gameza E.I.R.L & Remate \\
20 & Velarde Soncco, Marco Antonio & Remate \\
21 & Cuayla Cruz, Edwinaldo & Remate \\
22 & Comercial Ayde E.I.R.L. & Remate \\
23 & Asencio Coaguila, Richard & Remate \\
24 & Servimediq E.I.R.L & No adjudicado \\
25 & Parillo Laquise, Rocio & Remate \\
\hline & & 25 \\
\hline
\end{tabular}

Fuente: Promotora Opción S.A. EAFC

\section{RESULTADOS}

Respecto al estado de conocimiento del financiamiento por fondos colectivos, el $20 \%$ indica que fue casi siempre; el $16 \%$ manifiesta que siempre y el $52 \%$ manifiesta que algunas veces y el $12 \%$ casi nunca. Se observa que no se conoce mucho sobre el financiamiento de fondos colectivos, debiéndose efectuar una mayor difusión.

Según si se difunde las bondades del sistema para la adquisición de activos; el $48 \%$ indica que algunas veces; el $24 \%$ manifiesta que casi siempre; el $16 \%$ indica que siempre; el $8 \%$ manifiesta que casi nunca y el $4 \%$ manifiesta que nunca (Tabla 01). Se puede apreciar que aún falta difundir las bondades que ofrece el sistema de fondos colectivos, debiéndose efectuar la difusión necesaria para un mayor conocimiento.

Tabla 1: Se difunde las bondades del sistema para la adquisición de activos.

\begin{tabular}{lrr}
\hline & $\mathbf{n}$ & $\mathbf{\%}$ \\
\hline Nunca & 1 & $4.0 \%$ \\
Casi nunca & 2 & $8.0 \%$ \\
Algunas veces & 12 & $48.0 \%$ \\
Casi siempre & 6 & $24.0 \%$ \\
Siempre & 4 & $16.0 \%$ \\
\hline Total & 25 & $100.0 \%$ \\
\hline
\end{tabular}

Fuente: Cuestionario elaborado, 2016. 
Sobre si considera que el periodo de duración del contrato se adecua a sus necesidades; el $60 \%$ manifiesta que algunas veces; el $20 \%$ indica que casi nunca; el $12 \%$ indica que casi siempre y el $8 \%$ manifiesta que nunca. De lo que se tiene, falta mejorar los plazos de duración del contrato de acuerdo a las necesidades de los usuarios. En lo relacionado a si considera aceptable el valor de la cuota mensual; el $44 \%$ indica que casi nunca; el $28 \%$ manifiesta que algunas veces; el $20 \%$ indica que casi siempre y el $8 \%$ manifiesta que nunca. Se entiende que aún falta mejorar el valor de la cuota mensual de acuerdo a la disponibilidad de los usuarios. Respecto a si considera que puede dejar como garantía el bien financiado, el $40 \%$ manifiesta que nunca; el $28 \%$ indica que casi nunca; el $20 \%$ indica que casi siempre y el $12 \%$ manifiesta que algunas veces. De lo que se aprecia que los representantes de las diferentes empresas no consideran necesario dejar como garantía el bien financiado.

Sobre si el nivel de las aportaciones está dentro del valor promedio del mercado; el $60 \%$ manifiesta que algunas veces; el $8 \%$ indica que casi siempre; el $8 \%$ manifiesta que casi nunca; el $4 \%$ manifiesta que siempre y el $4 \%$ indica que nunca. Se entiende que falta mejorar el nivel de las aportaciones de acuerdo al promedio del mercado.

En lo relacionado a si tiene conocimiento de que podría dejar otro bien como garantía del bien financiado; el $4 \%$ manifiesta algunas veces; el $28 \%$ indica que casi siempre; el $16 \%$ indica que casi nunca; el $12 \%$ manifiesta que nunca; y el $4 \%$ manifiesta que siempre. Se observa que aún se desconoce sobre la posibilidad de dejar otro bien en garantía del bien financiado, debiendo efectuar la difusión necesaria para conocimiento de los usuarios.

En relación a si considera que el certificado de compra se entrega de manera oportuna; el $52 \%$ manifiesta que algunas veces; el $20 \%$ indica que casi siempre; el $12 \%$ indica que casi nunca; el $8 \%$ manifiesta que nunca y el $8 \%$ manifiesta siempre. Falta mejorar la entrega oportuna de los certificados de compra, debiendo implementar los correctivos necesarios.

Respecto a si considera que los contratos favorecen a las empresas; el $48 \%$ manifiesta que algunas veces; el $40 \%$ manifiesta que nunca; el $8 \%$ indica casi siempre; el $4 \%$ manifiesta que siempre. De lo que se entiende que aún falta difundir sobre el financiamiento con fondos colectivos ya que muy pocas empresas hacen uso de ellos, en la que indican que si favorecen al crecimiento de la empresa.

Con respecto a si las actividades de financiamiento se producen de manera regular de acuerdos a los requerimientos, el $36 \%$ manifiesta que nunca; el $28 \%$ indica que casi nunca; el $24 \%$ manifiesta que algunas veces; el $8 \%$ indica que casi siempre; y el $4 \%$ manifiesta que siempre (Tabla 02 ).

\begin{tabular}{lrr}
\multicolumn{4}{l}{ Tabla 02: Regularidad De Contratos } \\
\hline & $\mathbf{n}$ & $\mathbf{\%}$ \\
\hline Nunca & 9 & $36.0 \%$ \\
Casi nunca & 7 & $28.0 \%$ \\
Algunas veces & 6 & $24.0 \%$ \\
Casi siempre & 2 & $8.0 \%$ \\
Siempre & 1 & $4.0 \%$ \\
\hline Total & 25 & $100.0 \%$ \\
\hline Fuente: Cuestionario elaborado, 2016.
\end{tabular}

Se aprecia que falta mayor difusión relacionada al financiamiento de los fondos colectivos de acuerdo a los requerimientos de los asociados.

Sobre si considera que con esta opción de compra puede mejorar su rentabilidad; el $40 \%$ indica que algunas veces; el $32 \%$ manifiesta que casi siempre; el $20 \%$ manifiesta casi nunca y el $8 \%$ indica que 
siempre. Se entiende que efectivamente la mayoría de estas empresas acogidas a esta fuente de financiamiento indican que ha mejorado su rentabilidad. Así también el $36 \%$ indica que casi nunca; el $32 \%$ manifiesta que algunas veces; el $20 \%$ manifiesta que nunca y el $12 \%$ indican que casi siempre el sistema satisface sus expectativas.

En lo relacionado a si los bienes a adjudicar son exhibidos para conocimiento de los usuarios; el $40 \%$ indica que algunas veces; el $28 \%$ manifiesta que casi siempre; el $20 \%$ indica que siempre y el $12 \%$ manifiesta que casi nunca. De lo que se puede apreciar que en su mayoría los bienes si son exhibidos para conocimiento y apreciación de los usuarios.

En lo relacionado a si considera que ha mejorado la producción de su empresa bajo esta modalidad de compra, el $8 \%$ indica que casi siempre; el $28 \%$ manifiesta que algunas veces; el $52 \%$ indica que casi nunca; el $8 \%$ manifiesta que siempre; el $4 \%$ manifiesta que nunca. Se aprecia que en algunos casos efectivamente ha mejorado la producción de la empresa bajo esta modalidad de financiamiento.

En la pregunta si aprecia un crecimiento en su empresa por la aplicación de este financiamiento; el $40 \%$ manifiesta que algunas veces; el $32 \%$ indica que casi nunca; el $16 \%$ manifiesta que siempre; el $8 \%$ indica que casi siempre y el $4 \%$ manifiesta que nunca. Se observa que, si existe un crecimiento, según las respuestas dadas indican que su empresa ha mejorado bajo esta modalidad de financiamiento (Tabla 03).

Tabla 03: Crecimiento de la empresa por la aplicación de este financiamiento.

\begin{tabular}{lrr}
\hline & $\mathbf{n}$ & $\mathbf{\%}$ \\
\hline Nunca & 1 & $4.0 \%$ \\
Casi nunca & 8 & $32.0 \%$ \\
Algunas veces & 10 & $40.0 \%$ \\
Casi siempre & 2 & $8.0 \%$ \\
Siempre & 4 & $16.0 \%$ \\
\hline Total & 25 & $100.0 \%$ \\
\hline Fuente: Cuestionario elaborado, 2016. & &
\end{tabular}

De los resultados obtenidos se puede apreciar que efectivamente el sistema de fondos colectivos como estrategia influye en la adquisición de activos de las empresas de la ciudad de Tacna en el 2016, por lo que es necesario efectuar una mayor difusión sobre esta modalidad de financiamiento que permite obtener la compra de activos bajo la modalidad de fondos colectivos los mismos que se encuentran de acuerdo al precio del mercado permitiendo un mayor desarrollo de las empresas que se han acogido a este financiamiento.

\section{DISCUSIÓN}

De los resultados obtenidos se puede apreciar que efectivamente el Sistema de Fondos Colectivos como estrategia influye de manera significativa en la adquisición de activos de las empresas de la ciudad de Tacna en el 2016, asimismo la falta de difusión de información sobre esta modalidad de autofinanciamiento; también favorece al desarrollo, ahorro de costos y rentabilidad empresarial, no generando sobreendeudamiento en el sistema financiero de las empresas de la ciudad de Tacna cuyos activos se han obtenido a través de una Administradora de Fondos Colectivos. Finalmente se observa un crecimiento y consolidación de las empresas que han adquirido bienes bajo esta alternativa, ya que los usuarios tienen la opción de elegir al proveedor de acuerdo a sus requerimientos de calidad y precios a fin de poder optimizar costos, siempre que la modalidad de adquisición sea a través de certificados de 
compra donde el asociado al momento de ser adjudicado selecciona el proveedor de su conveniencia. Salazar, O (2010), indica que es factible realizar un fondo colectivo de desarrollo inmobiliario en la ciudad de Quito, para mejorar un fondo colectivo de esta clase, el fondo colectivo de desarrollo inmobiliario brinda rentabilidades diferenciales al 3.50\% en promedio mensual que obtienen los inversionistas, administrativa y financieramente cuenta con una estructura organizacional adecuada, para el manejo de este tipo de fondos, en beneficio de las empresas, lo que se relaciona con el presente trabajo de investigación sobre el beneficio que se da a las empresas. Manrique, J (2013) indica que los fondos mutuos han permitido a varias corporaciones obtener financiamiento a un costo relativamente menor que en el sistema bancario tradicional. Estas afirmaciones tienen afinidad con el presente trabajo de investigación ya que toman en cuenta el uso de fondos colectivos para la financiación de la empresa. Isa N., Romani F., Romani J. (2013) propone que la industria de fondos de inversión especializados atiende la demanda de financiamiento por parte de la mediana empresa y que puede ser mucho más eficiente que el sistema bancario.

Se observa que los fondos colectivos influyen en el beneficio económico de las empresas de la ciudad de Tacna, observándose que falta mejorar los plazos relacionados a la duración del contrato, a fin de implementar bajo este sistema la adquisición de activos fijos. Se puede apreciar que los fondos colectivos influyen en la adquisición de bienes de las empresas de la ciudad de Tacna, observándose que falta difundir la posibilidad de seleccionar sus proveedores con los cuales puede adquirir sus bienes a fin de poder escoger la mejor opción, generando un ahorro para la empresa. A través de la gerencia se debe implementar las acciones necesarias a fin de poder difundir las bondades que ofrece el sistema de fondos colectivos como una fuente de financiamiento para mejorar el crecimiento y desarrollo de la empresa. Asimismo, se debe implementar a través de la administración, mejores posibilidades de pago y/o importe de las cuotas a fin de que sea más accesible para los usuarios poder adquirir sus bienes bajo esta modalidad de compra en la adquisición de activos fijos. Se deberá efectuar una mayor difusión sobre la posibilidad de las empresas a elegir los proveedores y mostrar una la relación referencial de los mismos, con los cuales puede adquirir sus bienes a fin de poder escoger la mejor opción, generando un ahorro para la empresa e implementar a través de la administración, campañas de difusión sobre esta fuente de financiamiento para la adquisición de bienes activos fijos y poder mejorar el crecimiento de la empresa, permitiendo mejorar su rentabilidad.

\section{REFERENCIAS BIBLIOGRÁFICAS}

Hernández, R., Fernández, C. y Baptista, P. (2010). Metodología de la Investigación. México: Mc. Graw Hill.

Hirache, L. (2014). Adquisición de activos a través de Empresas Administradoras de Fondos Colectivos (EAFC). Actualidad Empresarial N 307-Segunda Quincena. p. 11-14

Imbaquingo, K. (2008). Propuesta de un sistema integrado para la administración y control de activos fijos de propiedad del Municipio del Cantón Mejía. (Tesis de pregrado). Escuela Politécnica del Ejército.

Isa N., Romani F., Romani J. (2013) Fondos de inversión como alternativa de financiamiento para la mediana empresa (Tesis de pregrado). Universidad Peruana de Ciencias.

Ley 22014. Empresas administradores de Fondos colectivos se constituirán como sociedades anónimas Diario oficial el peruano. Lima. Perú. 05 de diciembre del 1977

Manrique, J (2013) Inversionistas Institucionales, Estructura de Financiamiento Empresarial (Tesis de Maestría). Universidad de San Martín de Porres. Lima

Montenegro J. (2013) "Efecto en los estados financieros por el registro del desmantelamiento o retiro futuro de un elemento del componente de las propiedades, planta y equipo para una empresa que se dedica a la distribución de combustible" (Tesis de pregrado) Universidad de San Carlos de Guatemala Facultad de Ciencias Económicas. 
Resolución de Superintendencia N00020-2014, Reglamento del Sistema de Fondos Colectivos y de sus Empresas Administradoras. Diario Oficial El Peruano, Perú, Lima, 21 de septiembre del 2014.

Salazar, O (2010). Estudio de factibilidad para el diseño de un fondo colectivo de desarrollo inmobiliario en la ciudad de Quito, para fondos Pichincha. (Tesis de pregrado). Universidad Politécnica Salesina Sede Quito.

Lira J. (23 de febrero del 2015) Activos del sistema de fondos colectivos crecieron 12\% en el 2014. Diario Gestión. Recuperado de: https://gestion.pe/mercados/smv-activos-sistema-fondoscolectivos-recieron-12-2014-2124253

Lira J. (18 de marzo 2016) ¿Qué debo tener en cuenta antes de ingresar a un fondo colectivo? Diario Gestión. Recuperado de: https://gestion.pe/tu-dinero/debo-cuenta-ingresar-fondo-colectivo114574

Effio F (2017) Todo sobre activo fijo, propiedad planta y equipo Recuperado de: https://www.google.com.pe/url?sa=t\&rct=j\&q=\&esrc=s\&source=web\&cd=3\&cad=rja\&uact=8 \&ved=0ahUKEwiOr8mn6MPXAhVMSiYKHZAWAk8QFggvMAI\&url=http\%3A\%2F\%2Faempres

Hernández, D. (2015). Fondos colectivos. Monografias.com. Obtenido de http://www.monografias.com/trabajos97/fondos-colectivos/fondos-colectivos.shtml

Sistema De Fondos Colectivos (12 de junio del 2017). Superintendencia de Mercados y valores Recuperado de:

http://www.smv.gob.pe/Frm_VerArticulo.aspx?data=A01ACE41D475DBBFE553A0B77266A32 748078C45EA48C40E6D141A10881827675A42524295E1665D3B1AB45880 